\title{
Evaluación de las fortalezas del carácter en estudiantes universitarios
}

\author{
Assessment of the strengths of character in university students
}

Recepción del artículo: 27-10-2020 | Aceptación del artículo: 21-12-2020

Ricardo Horacio, Cortese Universidad Abierta Interamericana Argentina ricardocortese@yahoo.com.ar

https://orcid.org/0000-0003-2268-0353

María Mercedes, Fernández Canales, Universidad Abierta Interamericana -

Argentina

mercedesfernandez.1987@gmail.com

https://orcid.org/0000-0002-1359-8414

Lucas, Giaquinto

Universidad Abierta Interamericana -

Argentina

giaquintolucas@yahoo.com.ar

https://orcid.org/0000-0001-9108-8658

Para referenciar este artículo:

Cortese, R. H., Fernández Canales, M. M. y Giaquinto, L. (2020). Evaluación de las fortalezas del carácter en estudiantes universitarios. Revista ConCiencia EPG, 5(2), 45-59. https://doi.org/10.32654/CONCIENCIAEP G.5-2.3

\section{Resumen}

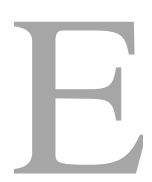
ste estudio identificó las fortalezas del carácter que predominaban en estudiantes universitarios de psicología e indagó si existían diferencias con individuos no universitarios, utilizando como medida de evaluación el IVyF (Cosentino \& Castro, 2008). Se seleccionó una muestra no probabilística compuesta por $\mathrm{N}=344$ participantes de ambos sexos, de entre 18 y 40 años de edad $(M=27,49$ DT $=6,76)$, residentes de Buenos Aires, la cual se subdividió en dos grupos: estudiantes universitarios de psicología $(\mathrm{N}=185)$ y no universitarios $(\mathrm{N}=159)$. Los resultados evidenciaron que las fortalezas que predominaron en los estudiantes fueron Integridad; Imparcialidad; Bondad; Apertura Mental; Perspectiva y Gratitud. Además, los universitarios presentaron mayor puntaje en comparación con los no universitarios en las fortalezas del carácter Apreciación, Imparcialidad, Persistencia, Creatividad, Amor, Gratitud, Liderazgo, Apertura Mental, Perspectiva, Humor y Amor por el saber; mientras que los no universitarios presentaron mayor puntaje en Inteligencia Social y Vitalidad.

Palabras Clave: psicología positiva - fortalezas del carácter - licenciatura en psicología. 
Abtract

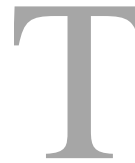

his research recognize the strengths of character that prevailed in university psychology students and looked into there were differences with non-university individuals, using the IVyF as an asessment measure (Cosentino \& Castro, 2008). A nonprobabilistic sample was selected, it was composed of $\mathrm{N}=344$ participants of both sexes, aged between 18 and $40(M=27.49$ $\mathrm{SD}=6.76)$, residents of Buenos Aires, which was subdivided into two groups: university students of psychology ( $\mathrm{N}=185)$ and nonuniversity students $(\mathrm{N}=159)$. The results showed that the strengths that predominated in the students were Integrity; Impartiality; Goodness; Mental opening; Perspective and Gratitude. Also, university students presented had higher scores compared to non-university students in haracter strengths Appreciation, Fairness, Persistence, Creativity, Love, Gratitude, Leadership, Open-mindedness, Perspective, Humor and Love of knowledge; while non-university students presented had higher scores in Social Intelligence and Vitality.

Key words: Positive Psychology - Strengths of Character - Bachelor's Degree in Psychology.

\section{Introducción}

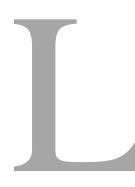

a carrera de psicología, al igual que otras carreras relacionadas con el área de la salud, implica para los estudiantes un gran sentido humanista y asistencial, ubicándola en un elemento de gran interés para el estudio del carácter.

A fin de adentrarse al tema, en la literatura científica se refleja que el carácter ha formado parte del discurso de la Psicología a principio del siglo pasado; pese a esto, su desarrollo fue recortado, dado que casi llegó a desaparecer como objeto pertinente de estudio para volver a resurgir a partir del presente siglo (McCullough \& Snyder, 2000; Cosentino, 2011).

Por otra parte, se encuentra que el estudio de las virtudes y fortalezas del carácter es un tema que ha sido abordado principalmente desde el enfoque salugénico de la Psicología Positiva, área que estudia el funcionamiento psíquico óptimo (Seligman, 2005), el cual ha sido dejado de lado por las corrientes tradicionales de psicología que se han orientado a reparar el daño (Seligman \& Csikszentmihalyi, 2000; Rivas, 2012).

De esta forma, se observa que desde el inicio de la Psicología Positiva se instaura una nueva perspectiva dentro del área de la salud mental, en la que los conceptos relacionados con las virtudes y fortalezas del carácter toman relevancia en el campo de la psicología científica. Específicamente, es cuando Peterson y Seligman (2004) desarrollan una clasificación de 6 virtudes que contienen 24 fortalezas del carácter ver Cuadro 1.

La Psicología Positiva considera a las virtudes y fortalezas del carácter como un concepto central dentro de su teoría, puesto que el carácter corresponde al estudio del área individual -que implica las 
características o rasgos de una persona, moralmente reconocidos y valorados- $\mathrm{y}$ ocupa un lugar de preponderancia sobre el área subjetiva -emociones positivas- $\mathrm{y}$ grupal -instituciones positivas- (Park \& Peterson, 2009).

Con respecto a la preponderancia del área individual por sobre la subjetiva y grupal, Cosentino (2011) explica que se debe a que las experiencias positivas subjetivas están sustentadas por el buen carácter y las instituciones son precisamente positivas porque están conformadas por individuos con buen carácter.

Según Peterson y Seligman (2004), el buen carácter se define como el producto de la interacción de determinados rasgos positivos e individuales que se denominan virtudes y fortalezas. Por lo tanto, el carácter es considerado un conjunto de rasgos o disposiciones positivas que son fundamentales para comprender el buen vivir psicológico y se estudia en tres niveles de abstracción.

El primer y máximo nivel de abstracción lo ocupan las virtudes, las cuales son los elementos centrales que han sido desarrollados por filósofos morales y pensadores religiosos. El segundo nivel corresponde a las fortalezas, que se consideran mecanismos psicológicos por medio de los que se muestran las virtudes. El tercer y último nivel se refiere a los temas situacionales, es decir, las circunstancias específicas que hacen que una persona muestre una determinada fortaleza en acción (Peterson \& Seligman, 2004; Castro, 2010; Cosentino, 2011).
Consecuentemente, Peterson y Seligman (2004) señalan que la jerarquía de las virtudes y fortalezas del carácter poseen tres niveles conceptuales.

- Virtudes: son las características nucleares y universales que se basan en la biología por medio del proceso evolutivo, el cual seleccionó determinadas características de excelencia, a fin de resolver cuestiones referidas a la supervivencia de la especie; éstas son: coraje, humanidad, justicia, sabiduría, templanza y trascendencia.

- Fortalezas: son reconocidas universalmente y corresponden a los componentes psicológicos o vías por las que se alcanza una virtud nuclear. Por ejemplo, la templanza se puede alcanzar a través de la clemencia, la humildad, la prudencia y/o la autorregulación.

- Temas situacionales -situational themes-: son los contextos o escenarios determinados que llevan a una persona a poner en marcha una o más fortalezas en determinada situación. De este modo, se demuestra que considerar los temas del contexto permite tener en cuenta la diversidad sociocultural en cómo las personas conciben cada fortaleza.

Peterson y Seligman

describen una serie de criterios para que a una fortaleza se considere como tal, algunos de ellos son: que permita lograr el desarrollo del bienestar y contribuir al afrontamiento de situaciones adversas; que colabore en encontrar el beneficio de ambas partes en una interacción; que sus expresiones sean acordes a las 
manifestaciones culturales $y$; a nivel individual se manifiesten a través de los sentimientos, pensamientos y de las acciones.

Por otra parte, Seligman (2005) diferencia a las fortalezas de las capacidades, estas últimas serían innatas y mayormente automáticas y, no implicarían voluntad.

Ahora bien, teniendo en cuenta a los sujetos que deciden enfocar su vocación hacia la carrera de Psicología, en la que se

Cuadro 1

Clasificación de las virtudes y fortalezas del carácter

\begin{tabular}{|c|c|c|}
\hline Virtudes & Características & Fortaleza \\
\hline \multirow{4}{*}{ Coraje } & Reúne 4 fortalezas emocionales & Valentía \\
\hline & que implican la consecución de & Persistencia \\
\hline & metas ante situaciones de & Integridad \\
\hline & dificultad, externa o interna & Vitalidad \\
\hline Humanidad & $\begin{array}{l}\text { Reúne } 3 \text { fortalezas } \\
\text { interpersonales que implican } \\
\text { cuidar y ofrecer amistad y cariño a } \\
\text { los demás. }\end{array}$ & $\begin{array}{l}\text { Amor } \\
\text { Bondad } \\
\text { Inteligencia social }\end{array}$ \\
\hline \multirow{3}{*}{ Justicia } & Reúne 3 fortalezas cívicas que & Ciudadanía \\
\hline & conllevan una vida en comunidad & Imparcialidad \\
\hline & saludable. & Liderazgo \\
\hline \multirow{5}{*}{$\begin{array}{l}\text { Sabiduría y } \\
\text { conocimiento }\end{array}$} & \multirow{5}{*}{$\begin{array}{l}\text { Reúne } 5 \text { fortalezas cognitivas que } \\
\text { implican la adquisición y el uso del } \\
\text { conocimiento. }\end{array}$} & Creatividad \\
\hline & & Curiosidad \\
\hline & & Apertura mental \\
\hline & & Amor por el saber \\
\hline & & Perspectiva \\
\hline \multirow{3}{*}{ Templanza } & \multirow{3}{*}{$\begin{array}{l}\text { Reúne } 4 \text { fortalezas que nos } \\
\text { protegen contra los excesos. }\end{array}$} & Clemencia y misericordia \\
\hline & & $\begin{array}{l}\text { Humildad/modestia } \\
\text { Prudencia }\end{array}$ \\
\hline & & Autorregulación \\
\hline Trascendencia & $\begin{array}{l}\text { Reúne } 5 \text { fortalezas que forjan } \\
\text { conexiones con la inmensidad del }\end{array}$ & $\begin{array}{l}\text { Apreciación de la belleza y } \\
\text { excelencia } \\
\text { Gratitud }\end{array}$ \\
\hline
\end{tabular}

pone a prueba la capacidad de contención, sostén, resolución, acompañamiento, orientación, reconocimiento de recursos de aquellas personas que poseen algún tipo de conflicto o patología que les genera una ruptura en su cotidianeidad, resulta relevante conocer las fortalezas de carácter que poseen los estudiantes, ya que resultarán una herramienta a la hora de abordar las diversas problemáticas con las que se enfrentarán como futuros profesionales dentro del campo de la salud mental. 


\section{universo y proveen de significado la vida. \\ Esperanza \\ Humor \\ Espiritualidad}

Fuente: Seligman \& Peterson (2004); Cosentino (2010).

En Argentina los autores que más se han enfocado en el estudio de las virtudes y fortalezas del carácter, tomando como punto de partida los aportes de Seligman y Peterson (2004), han sido Cosentino y Castro; es por esto por lo que, a continuación, se describen los hallazgos acerca del constructo en cuestión.

Cosentino (2010) fue quien llevó a cabo la tarea de evaluar las virtudes y fortalezas en población adulta de ambos sexos de habla hispana, con el fin de identificar las propiedades psicométricas del Inventario de virtudes $\mathrm{y}$ fortalezas [IVyF]. De esta manera, a partir del análisis factorial, llego a la conclusión de que el IVyF era una medida breve apropiada para la evaluación rápida de las fortalezas humanas según la clasificación del carácter de Peterson y Seligman (2004). Además, los análisis psicométricos preliminares evidenciaron que los puntajes del IVyF demostraron una adecuada confiabilidad y validez de constructo.

Posteriormente, Cosentino y Castro (2012) se plantearon seguir indagando en el constructo de virtudes y fortalezas del carácter, por un lado estudiando las diferencias de los rasgos positivos entre estudiantes universitarios militares $\mathrm{y}$ civiles, y entre cadetes de primero y último año de una academia militar; y por el otro, buscando la relación entre los rasgos positivos y los rendimientos académicos y militares de dichos cadetes siguiendo la clasificación de rasgos positivos de Peterson y Seligman (2004). A fin de lograr sus objetivos, administraron un autoinforme de rasgos positivos de 24 ítems, una medida de deseabilidad social y las calificaciones objetivas de los rendimientos académicos y militares. Los resultados evidenciaron que los estudiantes militares poseían mayores niveles de la fortaleza espiritualidad que los estudiantes civiles. A la vez, fueron distintas las relaciones entre las fortalezas y los rendimientos para primero y último año de cursada militar, en especial en el último curso, los cadetes con mejor adaptación a la academia obtuvieron mayores niveles de la fortaleza persistencia, en comparación con los cadetes de bajos rendimientos del mismo año de estudios.

Con la intención de seguir avanzando en las propiedades psicométricas del instrumento IVyF para medir las 24 fortalezas del carácter propuestas por Peterson y Seligman (2004), Cosentino y Castro (2015) partieron de la afirmación que, a diferencia de otros cuestionarios para medir las fortalezas de esa clasificación que están compuestos por decenas de ítems, el IVyF era un instrumento de medición breve (24 ítems) y volvieron a enfocarse en la validez del mismo. Para esto, primero evidenciaron que el IVyF se asociaba a medidas de constructos relevantes como satisfacción 
con la vida, las dimensiones de personalidad del modelo Big Five, y deseabilidad social. Luego, hallaron fuertes asociaciones entre auto-puntuación y puntuación realizada por un observador. Considerando estos resultados, los autores demostraron que fueron similares a los obtenidos en los cuestionarios homólogos. Por lo tanto, concluyeron que el IVyF presentó no solo adecuada confiabilidad, sino también adecuada validez.

Finalmente, en una investigación reciente, Castro y Cosentino (2017) exploraron la validez cultural del IVyF, encontrando que en adultos argentinos $($ edad media $=41,4$ años - DT $=13.1)$ de ambos sexos, las fortalezas más frecuentes fueron la integridad, la persistencia, la bondad y el amor; mientras que no encontraron diferencias según sexo y edad.

Teniendo en cuenta otras investigaciones científicas, se rastrearon dos estudios que también han indagado en las virtudes y fortalezas del carácter en población universitaria, dentro y por fuera de la población argentina. Por ende, en lo que sigue se revelan mencionados hallazgos científicos.

Uno de los hallazgos fue realizado por Candás, Mariñelarena y Martínez (2011), quienes exploraron las virtudes y fortalezas del carácter de 50 estudiantes universitarios de las carrera de Psicología y Ciencias de la educación de la Facultad de Ciencias Humanas de la Universidad Nacional de San Luis (Argentina), por medio del IVyF (Cosentino \& Castro, 2008). Los resultados mostraron que las fortalezas predominantes de la muestra total fueron: bondad, integridad, imparcialidad, humildad, apreciación de la belleza y la excelencia, gratitud, ciudadanía, prudencia, apertura mental, inteligencia social, valentía y persistencia; mientras que las que se presentaron en menor medida fueron: autorregulación y amor por el saber.

Por último, se encuentra el estudio de González (2015) llevado a cabo con estudiantes de Psicología de la Universidad de Rafael Urdaneta (Venezuela) que completaron el Inventario de Fortalezas del Carácter [VIA] de Peterson y Seligman (2004), en la versión traducida al español por Vázquez y Hervás (2002). En el caso específico de esta población universitaria, se evidenció que la media más alta fue para la fortaleza autocontrol, seguida por la esperanza, la honestidad, la gratitud y la apertura mental y las medias más bajas se presentaron en liderazgo, creatividad, civismo, humildad y amor/apego.

En función de lo expuesto, este estudio se propone identificar las fortalezas del carácter que predominan en estudiantes universitarios de la Licenciatura en Psicología de una institución privada de Buenos Aires e indagar si existen diferencias significativas con individuos no universitarios, utilizando como medida de evaluación el IVyF (Cosentino \& Castro, 2008), debido a que ha demostrado ser un instrumento con adecuadas propiedades psicométricas en la población argentina.

Therefore, in the communicative approach, it is fundamental to transmit to the student the contents and functions of 
the English language. That is, the real situations and places in which this language needs to be applied, for example: banks, airports, supermarkets, cinemas, and laundromats, among other places. That is why, the content that are given and taught, should correspond with the purpose of daily English use like buying clothes, food and other needs. This being said, it allows for the development of specific vocabulary to ask for and give information according to the context in which the student finds themselves.

\section{Método}

Diseño. Estudio de enfoque cuantitativo con alcance descriptivo y comparativo, en secuencia de tiempo transversal (Hernández, Fernández \& Baptista, 2006).

Participantes. Selección de una muestra no probabilística, intencional, compuesta por $\mathrm{N}=344$ sujetos de ambos sexos (Femenino 72,7\% - Masculino $27,3 \%$ ), de entre 18 y 40 años de edad $(\mathrm{M}=27,49-\mathrm{DT}=6,76)$, residentes de Bs. As. - ver Tabla 1. La muestra se subdividió en dos grupos:

Grupo A: aquellos sujetos que al momento de responder la encuesta eran estudiantes regulares de la carrera universitaria de Psicología; compuesta por $\mathrm{N}=185$ de ambos sexos (Femenino 79,5\% Masculino 20,5\%) de entre 18 y 40 años de edad (M=26,17 - DT=6,40).

Grupo B: aquellos sujetos que al momento de responder la encuesta no se encontraban estudiando en un establecimiento universitario; compuesta por $\mathrm{N}=$ 159 de ambos sexos (Femenino $64,8 \%$ - Masculino 35,2\%) de entre 18 y 40 años de edad ( $M=29,03-$ $\mathrm{DT}=6,87)$.

Instrumentos. Cuestionario sociodemográfico ad hoc, realizado para los fines de la investigación.

Inventario de virtudes y fortalezas del carácter IVyF. (Cosentino \& Castro, 2008)i. Inventario desarrollado para evaluar las 6 virtudes y 24 fortalezas del carácter según la clasificación de Peterson y Seligman (2004). Incluye 24 ítems bipolares con 5 opciones de respuesta tipo Likert, para que los encuestados indiquen en qué grado se parecen más a dos autodescripciones enfrentadas, una de ausencia de la fortaleza y la otra de presencia. La puntuación de cada ítem va de 1 (Soy muy parecido a la 1a persona) a 5 (Soy muy parecido a la $2^{a}$ persona), correspondiendo el puntaje más alto, a mayor presencia de la fortaleza. La mitad de los ítems se puntúan de forma directa. Los puntajes de las escalas de virtudes se obtienen promediando el puntaje de los ítems que corresponden a cada virtud.

Procedimiento. El relevamiento de datos se realizó en la Universidad Abierta Interamericana, Facultad de Psicología y Relaciones Humanas, luego se contactó a sujetos no universitarios por medio de los estudiantes de la Cátedra de Psicopatología. Se administró, bajo la modalidad de lápiz y papel, un breve cuestionario sociodemográfico realizado para los fines de esta investigación y el Inventario de virtudes y fortalezas del carácter. La participación en el estudio fue anónima y voluntaria, se entregó a cada participante que aceptó ser 
parte de ella un consentimiento informado y la información necesaria para completar las medidas de evaluación.

Análisis de datos. Para el análisis y procesamiento de los datos se utilizó el programa informático estadístico SPSS versión 20.0 en español con un nivel de significación de 0.05, empleando

Tabla 2

Descriptivos datos generales

\begin{tabular}{llll}
\hline \multirow{2}{*}{ Variables } & \multicolumn{1}{c}{$\mathbf{N = 3 4 4}$} & Frecuencia & Porcentaje \\
\hline \multirow{2}{*}{ Sexo } & Femenino & 250 & 72,7 \\
& Masculino & 94 & 27,3 \\
\hline \multirow{2}{*}{ Residencia } & CABA & 71 & 20,6 \\
& GBA & 273 & 79,4 \\
\hline \multirow{2}{*}{ Estado Civil } & Soltero/a & 142 & 41,3 \\
& En pareja & 122 & 35,5 \\
& Casado/a & 51 & 14,8 \\
\multirow{2}{*}{ Asistencia psicológica } & Separado/a & 19 & 5,5 \\
& Casado/a & 10 & 2,9 \\
\hline \multirow{2}{*}{ Grupo } & No & 86 & 25 \\
& Universitario & 185 & 75 \\
\hline
\end{tabular}

\section{Descripción de las fortalezas del carácter} - IVyF.

El análisis descriptivo de las fortalezas del carácter evidenció que en la muestra general predominaron las fortalezas Integridad (M=4,27), seguida por Bondad $(M=4,06)$, mientras que el menor puntaje se presentó en Espiritualidad $(M=2,91)$ - ver Tabla 3.

En el Grupo A, es decir el correspondiente a los estudiantes universitarios de la carrera de Psicología, se presentó un puntaje medio mayor en las estadísticos descriptivos y análisis de diferencia de grupos.

\section{Resultados}

\section{Descripción de datos generales}

Por último, en el Grupo B, es decir el correspondiente a los individuos no universitarios, se presentó un puntaje medio mayor en las fortalezas del carácter Integridad $(M=4,26)$; Vitalidad $(M=4,06)$ y Bondad $(M=4,04)$ y los menores puntajes se presentaron en Amor por el saber $(\mathrm{M}=2,92)$ y Autorregulación $(\mathrm{M}=2,93)$ - ver Tabla 5.

fortalezas del carácter Integridad $(\mathrm{M}=4,27)$; Imparcialidad $(\mathrm{M}=4,12)$; Bondad $(\mathrm{M}=4,07)$; Apertura Mental $(\mathrm{M}=4,05)$; Perspectiva $(\mathrm{M}=4,05)$ y Gratitud $(\mathrm{M}=4,01)$ y el menor puntaje se presentó en Espiritualidad $(\mathrm{M}=2,83)$ - ver Tabla 4 . 
Tabla 3

Descriptivos Fortalezas muestra general

\begin{tabular}{lllll}
\hline \multicolumn{1}{c}{ Fortalezas } & Media & Mediana & Moda & DT \\
\hline Apreciación & 3,82 & 4 & 4 & 1 \\
Imparcialidad & 3,82 & 4 & 5 & 1,15 \\
Persistencia & 3,79 & 4 & 4 & 1,09 \\
Creatividad & 3,65 & 4 & 4 & 1,06 \\
Amor & 3,47 & 4 & 4 & 1,18 \\
Autorregulación & 3,02 & 3 & 3 & 1,17 \\
Gratitud & 3,77 & 4 & 4 & 1,15 \\
Liderazgo & 3,74 & 4 & 4 & 1,12 \\
Apertura Mental & 3,94 & 4 & 4 &, 99 \\
Inteligencia Social & 3,85 & 4 & 4 & 1,09 \\
Clemencia & 3,66 & 4 & 4 & 1,04 \\
Espiritualidad & 2,91 & 3 & 3 & 1,19 \\
Ciudadanía & 3,55 & 4 & 4 & 1,08 \\
Valentía & 3,55 & 4 & 4 & 1,05 \\
Curiosidad & 3,77 & 4 & 4 & 1,10 \\
Bondad & 4,06 & 4 & 5 &, 99 \\
Esperanza & 3,58 & 4 & 4 & 1,08 \\
Integridad & 4,27 & 4 & 4 &, 75 \\
Perspectiva & 3,94 & 4 & 4 &, 82 \\
Prudencia & 3,81 & 4 & 4 &, 95 \\
Humor & 3,53 & 4 & 4 & 1 \\
Humildad Modestia & 3,65 & 4 & 3 & 1,07 \\
Amor por el saber & 3,34 & 3 & 4 & 1,14 \\
Vitalidad & 3,85 & 4 & & \\
\hline & & & 4 & \\
\hline
\end{tabular}

\section{Tabla 4}

Descriptivos Fortalezas estudiantes universitarios

\begin{tabular}{lllll}
\hline \multicolumn{1}{c}{ Fortalezas (N= 185) } & Media & Mediana & Moda & DT \\
& & 4 & 4 &, 89 \\
\hline Apreciación & 3,94 & 4 & 5 &, 94 \\
Imparcialidad & 4,12 & 4 & 5 & 1,04 \\
Persistencia & 3,92 & 4 & 4 & 1 \\
Creatividad & 3,77 & 4 & 4 & 1,03 \\
Amor & 3,75 & 3 & 3 & 1,19 \\
Autorregulación & 3,09 & 4 &, 92 \\
Gratitud & 4,01 & 4 & & \\
\hline
\end{tabular}




\begin{tabular}{lllll}
\hline Liderazgo & 3,91 & 4 & 4 & 1,08 \\
Apertura Mental & 4,05 & 4 & 5 &, 95 \\
Inteligencia Social & 3,74 & 4 & 5 & 1,19 \\
Clemencia & 3,72 & 4 & 4 & 1 \\
Espiritualidad & 2,83 & 3 & 3 & 1,21 \\
Ciudadanía & 3,55 & 4 & 4 & 1,11 \\
Valentía & 3,62 & 4 & 4 & 1,09 \\
Curiosidad & 3,69 & 4 & 4 & 1,10 \\
Bondad & 4,07 & 4 & 5 & 1,05 \\
Esperanza & 3,54 & 4 & 4 & 1,11 \\
Integridad & 4,27 & 4 & 4 &, 73 \\
Perspectiva & 4,05 & 4 & 4 &, 77 \\
Prudencia & 3,77 & 4 & 4 &, 92 \\
Humor & 3,74 & 4 & 4 &, 95 \\
Humildad Modestia & 3,65 & 4 & 3 & 1,02 \\
Amor por el saber & 3,70 & 4 & 4 & 1 \\
Vitalidad & 3,67 & 4 & 4 & 1,10 \\
\hline
\end{tabular}

Tabla 5

Descriptivos Fortalezas No universitarios

\begin{tabular}{lcccc}
\hline \multicolumn{1}{c}{ Fortalezas (N=159) } & Media & Mediana & Moda & DT \\
& & & 3 & 1,10 \\
\hline Apreciación & 3,67 & 4 & 5 & 1,26 \\
Imparcialidad & 3,48 & 4 & 4 & 1,14 \\
Persistencia & 3,65 & 4 & 3 & 1,10 \\
Creatividad & 3,51 & 4 & 3 & 1,25 \\
Amor & 3,15 & 3 & 3 & 1,15 \\
Autorregulación & 2,93 & 3 & 4 & 1,33 \\
Gratitud & 3,49 & 4 & 3 & 1,15 \\
Liderazgo & 3,55 & 4 & 4 & 1,03 \\
Apertura Mental & 3,81 & 4 & 4 &, 96 \\
Inteligencia Social & 3,99 & 4 & 4 & 1,09 \\
Clemencia & 3,59 & 4 & 3 & 1,16 \\
Espiritualidad & 3,00 & 3 & 3 & 1,06 \\
Ciudadanía & 3,54 & 4 & 3 & 1,01 \\
Valentía & 3,47 & 3 & 4 & 1,09 \\
Curiosidad & 3,87 & 4 & 5 &, 91 \\
Bondad & 4,04 & 4 & 4 & 1,03 \\
Esperanza & 3,63 & 4 & 5 &, 77 \\
Integridad & 4,26 & 4 & 4 &, 87 \\
Perspectiva & 3,80 & 4 & &
\end{tabular}




\begin{tabular}{lllll}
\hline Prudencia & 3,86 & 4 & 4 &, 98 \\
Humor & 3,28 & 3 & 3 & 1,01 \\
Humildad Modestia & 3,65 & 4 & 3 & 1,12 \\
Amor por el saber & 2,92 & 3 & 3 & 1,15 \\
Vitalidad & 4,06 & 4 & 5 &, 95 \\
\hline
\end{tabular}

\section{Análisis de diferencias de grupos}

Existieron

diferencias

estadísticamente significativas en 13 fortalezas del carácter entre el Grupo A, de estudiantes universitarios, y el grupo B, de no universitarios.

Los estudiantes universitarios presentaron mayor puntaje en comparación con los no universitarios en las fortalezas del carácter Apreciación ( $t=$

Tabla 6

Comparación entre grupos Universitarios y No universitarios
2.4; $p=.01)$, Imparcialidad ( $t=5.2 ; p=.00)$, Persistencia $(t=2.2 ; p=.02)$, Creatividad $(t=$ $2.2 ; p=.02)$, Amor ( $t=4.7 ; p=.00)$, Gratitud $(t=4.1 ; p=.00)$, Liderazgo $(t=3.0 ; p=.00)$, Apertura Mental $(t=2.1 ; \quad p=.02)$, Perspectiva $(t=2.8$; $p=.00)$, Humor $(t=2.4$; $p=.01)$, Amor por el saber $(t=6.6 ; p=.00)$; mientras que los No universitarios presentaron mayor puntaje en Inteligencia Social $(t=-2.1 ; p=.03)$ y Vitalidad $(t=-3.5$; $p=.00$ ) - ver Tabla 6.

\begin{tabular}{llllllc}
\hline & \multicolumn{2}{l}{$\begin{array}{l}\text { Universitarios } \\
(\mathbf{n = 1 8 5 )}\end{array}$} & \multicolumn{7}{l}{ No Universitarios } \\
Media & $\boldsymbol{D T}$ & Media & $\boldsymbol{D T}$ & $\boldsymbol{t}^{*}$ & Sig. \\
\hline Variables & 3,9 &, 89 & 3,6 & 1,1 & 2,4 &, 01 \\
\hline Apreciación & 4,1 &, 94 & 3,4 & 1,2 & 5,2 &, 00 \\
Imparcialidad & 3,9 & 1 & 3,6 & 1,1 & 2,2 &, 02 \\
Persistencia & 3,7 & 1 & 3,5 & 1,1 & 2,2 &, 02 \\
Creatividad & 3,7 & 1 & 3,1 & 1,2 & 4,7 &, 00 \\
Amor & 3,0 & 1,1 & 2,9 & 1,1 & 1,2 &, 20 \\
Autorregulación & 4,0 &, 92 & 3,4 & 1,3 & 4,1 &, 00 \\
Gratitud & 3,9 & 1 & 3,5 & 1,1 & 3,0 &, 00 \\
Liderazgo & 4,0 &, 95 & 3,8 & 1 & 2,1 &, 02 \\
Apertura mental & 3,7 & 1,1 & 3,9 &, 96 & $-2,1$ &, 03 \\
Inteligencia Social & 3,7 & 1 & 3,5 & 1 & 1,1 &, 26 \\
Clemencia & 2,8 & 1,2 & 3 & 1,1 & $-1,3$ &, 18 \\
Espiritualidad & & & &
\end{tabular}




\begin{tabular}{lllllll} 
Ciudadanía & 3,5 & 1,1 & 3,5 & 1 &,- 09 &, 92 \\
Valentía & 3,6 & 1 & 3,4 & 1 & 1,2 &, 20 \\
Curiosidad & 3,6 & 1,1 & 3,8 & 1 & $-1,5$ &, 12 \\
Bondad & 4,0 & 1 & 4 &, 91 &, 24 &, 80 \\
Esperanza & 3,5 & 1,1 & 3,6 & 1 &,- 76 &, 44 \\
Integridad & 4,2 &, 73 & 4,2 &, 77 &, 07 &, 94 \\
Perspectiva & 4,0 &, 77 & 3,8 &, 87 & 2,8 &, 00 \\
Prudencia & 3,7 &, 92 & 3,8 &, 98 &,- 85 &, 39 \\
Humor & 3,7 &, 95 & 3,2 & 1 & 4,2 &, 00 \\
Humildad/Modestia & 3,6 & 1 & 3,6 & 1,1 & 0,5 &, 95 \\
Amor por el saber & 3,7 & 1 & 2,9 & 1,1 & 6,6 &, 00 \\
Vitalidad & 3,6 & 1,1 & 4 &, 95 & $-3,5$ &, 00 \\
\hline
\end{tabular}

*Nota. Se empleó el análisis $t$ de Student.

\section{Discusión}

El objetivo de este estudio fue identificar las fortalezas del carácter que predominaban en estudiantes universitarios de la Licenciatura en Psicología de una institución privada de Buenos Aires utilizando como medida de evaluación el IVyF (Cosentino \& Castro, 2008), puesto que es un instrumento con adecuadas propiedades psicométricas en la población argentina (Cosentino, 2010; Cosentino \& Castro, 2015; Castro \& Cosentino, 2017). A partir de los resultados se evidenció que las fortalezas que predominaron con mayor frecuencia en los estudiantes fueron Integridad; Imparcialidad; Bondad; Apertura Mental; Perspectiva y Gratitud, mientras que la de menor frecuencia fue Espiritualidad.

Adicionalmente, se realizó un análisis comparativo para indagar si existían diferencias significativas en las fortalezas del carácter entre participantes universitarios y no universitarios. El análisis estadístico demostró que los estudiantes universitarios presentaron un mayor puntaje en comparación con los no universitarios en las fortalezas del carácter Apreciación; Imparcialidad; Persistencia; Creatividad; Amor; Gratitud; Liderazgo; Apertura Mental; Perspectiva; Humor y Amor por el saber; mientras que los no universitarios presentaron mayor puntaje en Inteligencia Social y Vitalidad.

Los resultados de este estudio indicarían que los estudiantes de psicología tienden a: ser coherentes con los valores que poseen; hacer juicios sociales equitativos; poseer una orientación hacia los demás con un tono afectivo que impulsa comportamientos de asistencia sin fines utilitarios; buscar activamente alternativas para modificar el punto de vista; poseer el 
conocimiento y la capacidad de dar consejos sobre la vida; sentir y expresar agradecimiento ante un beneficio tangible o intangible. Por otra parte, presentaron una menor tendencia a emplear la fortaleza Espiritualidad, referida a las creencias y prácticas que se relacionan con una dimensión trascendente, es decir, no física, en la que la vida posee un propósito o significado superior (Petersen \& Seligman, 2004; Cosentino, 2011).

Asimismo, se hallaron diferencias entre los estudiantes universitarios y los participantes no universitarios respecto a las fortalezas del carácter. Los estudiantes universitarios tendieron a presentar una mayor capacidad para realizar juicios sociales objetivos; dirigir en armonía grupos hacia el éxito; mantenerse cerca de sus afectos; reflexionar de manera profunda acerca de la vida; poseer ideas originales, útiles y adaptativas; buscar enfoques alternativos; interesarse por adquirir nuevos conocimientos $y$ habilidades; sentir y expresar agradecimiento; presentar una postura calma y risueña frente a la vida que permite ver el lado positivo ante las adversidades y mantener un buen estado de ánimo; encontrar, reconocer y sentir admiración o asombro hacia lo ilustre del entorno físico y social, y finalizar las tareas superando los obstáculos. Por su parte, los no universitarios tendieron a presentar mayor capacidad de razonar sobre la información emocional en las relaciones con los demás y de experimentar la vivencia subjetiva de sentirse vivos y con energía (Petersen \& Seligman, 2004; Cosentino, 2010).

Se destaca como limitaciones que la muestra fue no probabilística, hecho que impide que se generalicen los resultados en la población general; además, se trabajó en secuencia de tiempo transversal, por lo que sería importante evaluar en futuras líneas de investigación si las fortalezas del carácter varían a medida que se avanza en la carrera y no pudieron establecerse diferencias de sexo, ya que predominó el femenino por sobre el masculino. Finalmente, tomando el estudio de Castro y Cosentino (2012) sería relevante examinar las posibles relaciones entre el rendimiento académico y las fortalezas del carácter en los estudiantes universitarios.

\section{Conclusiones}

En conclusión, este estudio se desprendió del proyecto de investigación trienal de la Cátedra de Psicopatología de la Universidad Abierta Interamericana [UAI] e intenta ser una aproximación al estudio de los rasgos positivos del carácter, a fin de establecer un perfil en la población de alumnos/as de la Licenciatura de Psicología de la UAI.

\section{Referencias}

Candás, S. B., Mariñelarena-Dondena, L. \& Martínez-Núñez, V. A. (2011). Evaluación de las virtudes y fortalezas del carácter en estudiantes universitarios. En $V$ Congreso Marplatense de Psicología. La Psicología en el porvenir de la cultura. El semejante: entre el 
enemigo y el desamparado. Facultad de Psicología, Universidad Nacional de Mar del Plata.

Castro Solano, A. (2010). Ensayo: Psicología positiva: ¿Una nueva forma de hacer psicología? Revista de Psicología, 6(11), 113-131.

Castro Solano, A. \& Cosentino, A. C. (2017). Virtues and character strengths in Buenos Aires adult population. A study with a mixed emic/etic approach. Summa Psicológica UST, 14(1), 23-32.

Cosentino, A. C. \& Castro Solano, A. (2008). Inventario de virtudes y fortalezas. Manuscrito no publicado.

Cosentino, A. C. (2010). Assessment of human virtues and strengths in Hispanic language population. Psicodebate, 10, 53-71.

Cosentino, A. C. (2011). Fortalezas del carácter en militares argentinos [Doctoral dissertation, Universidad de Palermo].

Cosentino, A. C. \& Castro Solano, A. (2012). Character Strengths: A Study of Argentinean Soldiers. The Spanish Journal of Psychology, 15(1), 199215.

Cosentino, A. C. (2014). Character strengths: Measurement and studies in Argentina with military and general population samples. En A. Castro Solano (Ed.), Positive Psychology in Latin America (pp. 111-127). Springer Netherlands.
Cosentino, A. C. \& Castro Solano, A. (2015). IVyF: Validity of a measuring instrument of the Peterson and Seligman (2004) strengths of character classification. Psicodebate, 15(2), 99-122.

González Llovera, S. M. (2015). Fortalezas de carácter en estudiantes universitarios de psicología. Encuentro Educacional, 22(3), 419-431.

Hernández Sampieri, R., Fernández Collado, C. \& Baptista Lucio, P. (2006). Metodología de la investigación. McGraw-Hill.

McCullough, M. E. \& Snyder, C. R. (2000). Classical source of human strength: Revisiting an old home and building a new one. Journal of Social \& Clinical Psychology, 19, 1-10.

Park, N. \& Peterson, C. (2009). Strengths of character in schools. En R. Gilman, E. Huebner \& M. Furlong (Eds.), Handbook of positive psychology in schools (pp. 65-76). Routledge.

Peterson, C. \& Seligman, M.E.P. (2004). Character strengths and virtues: $A$ handbook and classification. Washington, DC: American Psychological Association: Oxford University Press.

Rivas de Mora, S. M. (2012). Fortalezas de carácter y resiliencia en estudiantes de Medicina de la Universidad de los Andes, Mérida, Venezuela [Tesis Doctoral Universidad Autónoma de Madrid]. España. 
Seligman, M. E. P. (2005). La Autentica Felicidad. Barcelona: Ediciones B, S.A.
Seligman, M. \& Csikszentmihalyi, M. (2000). Positive Psychology: An Introduction. American

\begin{abstract}
${ }^{i}$ A pedido de los autores del IVyF se incluye la nota de Copyright: Alejandro César Cosentino y Alejandro Castro Solano poseen el copyright del IVyF y su clave de corrección. Los siguientes son los límites del uso razonable o justo para el uso del IVyF. Se autoriza a usar el inventario IVyF tal cual está y sin ninguna garantía, en su formato original de papel y lápiz, para uso exclusivo en la investigación académica sin fines de lucro bajo el acuerdo con los autores del IVyF (tanto del IVyF como su clave de corrección) y se autoriza únicamente a la realización de las copias necesarias en papel para que los participantes de la investigación sin fines de lucro las completen. Sin embargo, no autorizamos la publicación del IVyF, ni su comercialización, ni su uso comercial ni su uso clínico, ni cualquier otro uso o fin que no sea utilizar el inventario tal cual está en el uso exclusivo para la investigación académica sin fines de lucro bajo acuerdo con los autores del inventario; ni tampoco damos autorización para la carga del IVyF en página web, alojamiento de internet o similar, ni su envío por correo electrónico, ni su distribución, copia, almacenamiento o publicación por cualquier medio ni bajo ningún concepto. La utilización del instrumento IVyF y su clave de corrección implican que la/s persona/s que desarrolla/n investigación y/o que lo utiliza/n acepta/n lo términos expuestos en este párrafo.
\end{abstract}

